\title{
Gene-expression profiling of localized prostate cancer: still miles to go before we sleep
}
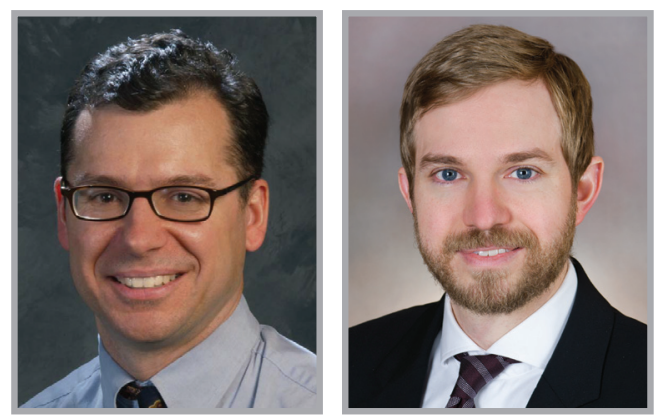

\author{
"Platforms that have recently \\ been developed for gene \\ discovery include PCR, \\ microarray and
} whole-genome sequencing."

Mark Garzotto ${ }^{* 1,2,3}$ \& Ryan P Kopp ${ }^{1,2}$

First draft submitted: 24 October 2015; Accepted for publication: 10 November 2015; Published online: 19 January 2016

Contemporary developments in genetic sequencing technologies and bioinformatics have led to the explosive growth of information available for clinical decision making in cancer [1]. In this context, the term 'genetic testing' covers an array of techniques including the analyses of human DNA, RNA or protein. Platforms that have recently been developed for gene discovery include polymerase chain reaction, microarray and whole-genome sequencing. Information garnered from genetic testing has been clearly demonstrated to improve cancer care in breast, colon, melanoma, leukemia, as well as other cancers. However, many challenges to informational implementation remain which include data computation, training physicians to interpret test results and in turn educate patients and their families. Competency in these areas requires a working knowledge of oncology, genetics and genetic counseling [2].

The spectrum of prostate cancer outcomes is dictated by its inherent inter- and intrapatient tumor heterogeneity. Characterization of prostate cancer through biomarker discovery beyond histologic grade determination has the potential to improve risk stratification and treatment selection in prostate cancer. The number of genetic tests available in prostate cancer has risen dramatically in the last decade and currently there are 52 available tests currently listed at the NIH Genetic Tumor Registry [3], thus rigorous and systematic evaluation of test performance is needed to prioritize test strategies. In this concise review, we will highlight some of the commonly used genetic/genomic and emerging tests for prostate cancer risk assessment.

\section{Multigene array tests for risk}

\section{stratification}

Through the use of pathway-based gene identification and genome-wide association strategies, several commercially available genomic tests assays have been developed for prostate cancer risk stratification.

\section{KEYWORDS}

- BRCA1 gene - BRCA2 gene - genetic testing $\bullet$ nomograms $\bullet$ prostate cancer - PTEN phosphatase

"Other univariate predictors of death included were prostate-specific antigen, Gleason and T-stage.” 


\section{"Early findings suggested germline BRCAT/2 mutations harbor an increased risk of developing prostate cancer particularly in younger patients.”}

The Prolaris ${ }^{\circledR}$ test (Myriad Genetics) is a multigene panel that is obtained from archived mRNA in formalin-fixed paraffin-embedded tissue. The test quantifies 46 genes which include 31 cell cycle progression (CCP) genes and 15 housekeeping genes to generate a CCP score [4]. The test is indicated for risk assessment of disease progression in low-risk prostate cancer patients who are candidates for active surveillance. In a study of 349 patients treated conservatively in the UK, a 1-unit increase in CCP was associated in univariate analysis with a twofold increase in prostate cancer death at 10 years $(\mathrm{p}<0.05)$. Other univariate predictors of death included were prostatespecific antigen (PSA), Gleason and T-stage [4]. In a US-based study by Cooperberg et al. CCP from prostatectomy specimens was compared with the CAPRA-S score using clinical factors alone [5]. In this comparison, the CCP was slightly better than the CAPRA-S alone (AUC 0.77 vs 0.73), but this difference was not statistically significant. The risk of progression was found to increase by 1.7 -fold for each unit increase in CCP; however, the median CCP was 0 and the population with a level of CCP of 1 or higher was only $3 \%$ of the entire study group. So although CCP was predictive of clinical outcome, it appeared to be most applicable to a very small subgroup of the cohort. Another limitation is that there has been no demonstration of clinical utility or benefit to date with the assay. However, in a survey of physicians, respondents reported that they would definitely modify their treatment choice only 3\% of the time [6].

Another test available for clinical risk assessment is the Oncotype Dx Genomic Prostate Score ${ }^{\circledR}$ (Genomic Health, Inc., CA, USA) which is indicated for consideration of active surveillance in low-risk patients. This assay measures levels of 12 cancer-related genes and five reference genes from mRNA in formalin-fixed paraffinembedded to generate a GPS score [7]. The GPS score (range: 1-100) is combined with clinical factors including Gleason score, PSA and tumor stage to give a GPS-modified risk assessment (low-intermediate or high risk). In a multicenter study of biopsy patients, GPS was able to predict the presence of favorable pathology in prostatectomy specimens [8]. For each 20-point increase in GPS, the risk of adverse pathology increased 1.9fold $(\mathrm{p}=0.003)$ in a model including individual age, PSA, Gleason and T-stage values. However, a significant limitation to this approach is that the GPS was not compared with any of the contemporary multifactor clinical nomograms commonly used in clinical practice. In terms of clinical utility, there are only very limited data to suggest that the GPS would be efficacious in a real-world setting. In an in silico modeling experiment of decision curve analysis, Klein et al. showed a theoretical reduction in overtreatment; however, these results must be interpreted with extreme caution as they can only be considered hypothesis generating. Lastly, it is unclear whether these data can be directly applied to a longitudinal active surveillance cohort as all patients in the study underwent a prostatectomy [8]. These lingering questions can only be answered through the performance of additional well-designed prospective trials.

\section{Single gene \& gene family markers} - BRCA1 (early onset) \& BRCA2 (early onset)

Early findings suggested germline BRCA1/2 mutations harbor an increased risk of developing prostate cancer particularly in younger patients [9,10], an association that is usually stronger in BRCA2, compared with BRCA1 [11]. Further retrospective data revealed prognostic associations with Gleason grade $\geq 8$, T3/T4 disease, nodal disease and distant metastases at diagnosis [12]. Additionally, BRCA carriers were nearly twice as likely (hazard ratio: 1.8 ) to die of prostate cancer, with a median survival of only 8.6 years, versus 15.7 years in noncarriers. A follow-up analysis in patients initially diagnosed with localized prostate cancer reported 10 -year metastasis-free survival of only $50 \%$ in carriers, versus $84 \%$ in noncarriers, in addition to cancerspecific survival of 61 and $85 \%$, respectively [13].

This mounting evidence provided impetus toward a screening trial specifically targeting $B R C A$ carriers [14]. Preliminary analysis from the IMPACT trial demonstrated that with a screening PSA threshold of $3.0 \mathrm{ng} / \mathrm{ml}$, the positive predictive value of biopsy among BRCA2 carriers was $48 \%$ (731 screened, 50 biopsied) and 37.5\% among BRCA1 carriers (791 screened, 48 biopsied). The $48 \%$ among $B R C A 2$ carriers is roughly two-times the positive predictive value of biopsy most population screening studies. Furthermore, the positive predictive value of biopsy for detecting intermediate or high-risk prostate cancer was significantly greater $(\mathrm{p}=0.04)$ among $B R C A 2$ carriers $(2.38 \%)$ versus controls $(0.71 \%)$ [14]

However, it is unclear how often sporadic prostate cancer may harbor $B R C A$ mutations. A multi-institutional collaborative performed integrative genomic studies of metastatic castrateresistant prostate cancer (mCRPC) from 
150 cases, finding that $23 \%$ of $\mathrm{mCRPC}$ have DNA repair pathway aberrations, and $8 \%$ with actionable pathogenic germline aberrations [15]. Analysis of aberrations included amplification, copy number alteration, mutation and fusion. Incidence of BRCA2 aberration was $13.3 \%$ ( $12.7 \%$ with loss, of which $90 \%$ were bi-allelic), while $B R C A 1$ was only $0.7 \%$. Germline $B R C A 2$ aberrations were present in $5 \%$. Somatic aberrations were substantial compared with a set of primary tumor samples, in which only two out of 109 had BRCA1/2 alterations [16]. Multiple patients with BRCA2 loss in the mCRPC cohort were previously enrolled in a trial evaluating poly(-ADP-ribose) polymerase inhibition for unselected $\mathrm{mCRPC}$ cases and were clinical responders to the drug, demonstrating $B R C A$ are clinically actionable and relevant mutations [17].

\section{- PTEN \& PI3K pathway}

Studies in the biology of prostate cancer identified PTEN and the PI3K pathway as having a role in development of mCRPC, and may be crucial when androgen receptor signaling is low [18]. Recent integrative genomic analysis demonstrated PTEN aberrations in $40.7 \%$ of mCRPC cases [15]. Including other members of the PI3K pathway (AKT1, PIK3CA/B), somatic alterations occurred in $49 \%$ of mCRPC. The incidence and prognosis of these alterations in localized disease is not well elucidated. One analysis of 612 radical prostatectomy specimens exhibited a PTEN deletion rate of $18.3 \%$. PTEN deletion was significantly associated with extracapsular extension, seminal vesical invasion and higher Gleason grade. Homozygous deletion was associated with the worst recurrence-free survival following surgery [19]. The clinical utility of these data has yet to be established through prospective evaluation.

There are many factors to be considered when evaluating a novel test for potential use in clinical practice. These include: are there logistical issues with test procedures (i.e., is the test material well preserved)? How well are clinical staff trained in the appropriate use and interpretation of the genetic test? How representative is the test cohort of the patient being tested? How did the assay improve patient care? To what degree was care improved? What was the cost of the test per unit of improved outcome? To assess the value in healthcare of genetic/genomic tests the Center on Disease Control Office of Public Health Genomics has commissioned an independent working group to regularly make comprehensive assessments of tests, placing them in one of three prioritization tiers [20]. These assessments are updated regularly and easily accessible to practicing clinicians. Currently no prostate cancer genetic test is listed in tier 1, a level at which it would be considered standard of care.

In conclusion, although the excitement surrounding genomic technology is leading to increased clinical utilization, we stress that more rigorous prospective clinical research is required to determine the clinical utility of these technologies in the management of prostate cancer when compared with current clinical methods of risk assessment.

\section{Disclaimer}

The content of this manuscript does not necessarily represent the views of the US Department of Veterans Affairs or the US Government.

\section{Financial \& competing interests disclosure}

This work was supported by VA Merit Review (M Garzotto) from the US Department of Veterans Affairs (Clinical Sciences Research). The authors have no other relevant affiliations or financial involvement with any organization or entity with a financial interest in or financial conflict with the subject matter or materials discussed in the manuscript apart from those disclosed.

No writing assistance was utilized in the production of this manuscript.

\section{References}

1 van Dijk EL, Auger H, Jaszczyszyn Y, Thermes C. Ten years of next-generation sequencing technology. Trends Genet. 30(9), 418-426 (2014).

2 Robson ME, Bradbury AR, Arun B et al. American Society of Clinical Oncology Policy Statement update: genetic and genomic testing for cancer susceptibility. J. Clin. Oncol. 33(31), 3660-3667 (2015).
3 NIH Genetic Tumor Registry (GTR). www.ncbi.nlm.nih.gov/gtr/

4 Cuzick J, Berney DM, Fisher G et al. Prognostic value of a cell cycle progression signature for prostate cancer death in a conservatively managed needle biopsy cohort. Br. J. Cancer 106(6), 1095-1099 (2012).

5 Cooperberg MR, Davicioni E, Crisan A, Jenkins RB, Ghadessi M, Karnes RJ. Combined value of validated clinical and genomic risk stratification tools for predicting prostate cancer mortality in a high-risk prostatectomy cohort. Eur. Urol. 67(2), 326-333 (2015).

6 Shore N, Concepcion R, Saltzstein D et al. Clinical utility of a biopsy-based cell cycle gene expression assay in localized prostate cancer. Curr. Med. Res. Opin. 30(4), 547-553 (2014).

7 Cullen J, Rosner IL, Brand TC et al. A Biopsy-based 17-gene genomic prostate score 


\section{EDITORIAL Garzotto \& Kopp}

predicts recurrence after radical prostatectomy and adverse surgical pathology in a racially diverse population of men with clinically low- and intermediate-risk prostate cancer. Eur. Urol. 68(1), 123-131 (2015).

8 Klein EA, Cooperberg MR, Magi-Galluzzi C et al. A 17-gene assay to predict prostate cancer aggressiveness in the context of Gleason grade heterogeneity, tumor multifocality, and biopsy undersampling. Eur. Urol. 66(3), 550-560 (2014).

9 Kote-Jarai Z, Leongamornlert D, Saunders E et al. BRCA2 is a moderate penetrance gene contributing to young-onset prostate cancer: implications for genetic testing in prostate cancer patients. Br. J. Cancer 105(8), 1230-1234 (2011).

10 Thompson D, Easton DF. Breast Cancer Linkage Consortium. Cancer Incidence in BRCA1 mutation carriers. J. Natl Cancer Inst. 94(18), 1358-1365 (2002).

11 Gallagher DJ, Gaudet MM, Pal P et al. Germline BRCA mutations denote a clinicopathologic subset of prostate cancer. Clin. Cancer Res. 16(7), 2115-2121 (2010).

12 Castro E, Goh C, Olmos D et al. Germline $B R C A$ mutations are associated with higher risk of nodal involvement, distant metastasis, and poor survival outcomes in prostate cancer. J. Clin. Oncol. 31(14), 1748-1757 (2013).

13 Castro E, Goh C, Leongamornlert D et al. Effect of $B R C A$ mutations on metastatic relapse and cause-specific survival after radical treatment for localised prostate cancer. Eur. Urol. 68(2), 186-193 (2015).

14 Bancroft EK, Page EC, Castro E et al. Targeted prostate cancer screening in $B R C A 1$ and $B R C A 2$ mutation carriers: results from the initial screening round of the IMPACT study. Eur. Urol. 66(3), 489-499 (2014).

15 Robinson D, Van Allen EM, Wu YM et al. Integrative clinical genomics of advanced prostate cancer. Cell 161(5), 1215-1228 (2015).

16 Barbieri CE, Baca SC, Lawrence MS et al. Exome sequencing identifies recurrent SPOP,
FOXA1 and MED12 mutations in prostate cancer. Nat. Genet. 44(6), 685-689 (2012).

17 Mateo J, Hall E, Sandhu S et al. Antitumour activity of the PARP inhibitor olaparib in unselected sporadic castration-resistant prostate cancer (CRPC) in the TOPARP trial. Ann. Oncol. 25(5), 1-41 (2014).

18 Carver BS, Chapinski C, Wongvipat J et al. Reciprocal feedback regulation of PI3K and androgen receptor signaling in PTENdeficient prostate cancer. Cancer Cell 19(5), 575-586 (2011).

19 Troyer DA, Jamaspishvili T, Wei W et al. A multicenter study shows PTEN deletion is strongly associated with seminal vesicle involvement and extracapsular extension in localized prostate cancer. Prostate 75(11), 1206-1215 (2015).

20 Dotson WD, Douglas MP, Kolor Ket al. Prioritizing genomic applications for action by level of evidence: a horizon-scanning method. Clin. Pharmacol. Ther. 95(4), 394-402 (2014). 